\title{
Pathophysiology of the lower urinary tract and CNS
}

\author{
Christopher Chapple, BSC, MD, DHC, FRCS (Urol), FEBU \\ Consultant Urological Surgeon, Sheffield Teaching Hospitals NHS Foundation Trust, Department of Urology, The Royal Hallamshire Hospital, Sheffield, UK
}

Cite as: Can Urol Assoc J 2011;5(5Supp|2):S126-127; D0I:10.5489/cuaj.11180

\section{Abstract}

A number of aspects of neural control are potentially important in the control of bladder function, including both sensory and motor and peripheral and central pathways. It is likely that a combination of disorders of both central and peripheral neural mechanisms is important in the genesis of urgency and the other symptoms of the overactive bladder $(\mathrm{OAB})$. Given the number of potential pathways involved, potential pharmacologic targets for OAB exist in the CNS (central nervous system; cerebral cortex, midbrain, spinal cord) and periphery (LUT; lower urinary tract). Antimuscarinics are still the mainstay of $O A B$ treatment, but there are also a number of other potentially efficacious drugs that may also provide benefit against the neurologic components of OAB. This review discusses the impact of neurological abnormalities on lower urinary tract symptoms and the potential for treatments targeting these pathways to improve symptoms.

ower urinary tract symptoms (LUTS) have a complex pathophysiology that includes both myogenic and neurologic aspects. This brief review discusses the impact of neurological abnormalities on LUTS and the potential for treatments targeting these pathways to improve symptoms.

\section{What is overactive bladder?}

Urinary symptoms are not disease specific. The storage symptom component of LUTS of overactive bladder (OAB) represents an empirical diagnosis used as the basis for initial management after assessing clinical history, physical findings, urinalysis, and other indicated evaluations.

Urgency is a sensation associated with abnormal bladder behaviour during the filling phase, which is difficult to define and explain to others who have not experienced it, but is quite distinct from normal urge (see Figure 1). ${ }^{1}$ Urgency is a pathological symptom, which leads to the rest of the urinary symptom complex - reduced intervoid interval, nocturia, incontinence and reduced urinary volume.

\section{Neurologic mechanisms underlying $O A B$}

While the precise role of neurologic abnormalities in $\mathrm{OAB}$ is not completely clear, those aspects that have been identified as potentially important in the control of bladder function are shown in Table 1. Importantly, one cannot focus solely on the periphery while ignoring the role of central neurologic aspects.

\section{Central}

The central nervous system (CNS) acting via a number of central nuclei - particularly in the limbic system under normal circumstances - provides negative feedback control of the pontine micturition centre. ${ }^{2}$ Failure of this normal processing may lead to a centrally mediated perception of urgency. It is likely that a combination of disorders of both central and peripheral neural mechanisms is important in the genesis of urgency and the other symptoms of OAB. Indeed, functional imaging studies have shown a correlation between central activity (particularly the limbic system and prefrontal cortex) and urgency. ${ }^{3,4}$

\section{Peripheral}

Neurogenic mechanisms of urinary symptoms in the periphery may also have a number of sources. Bladder distention may directly activate the suburothelial neural plexus. In addition, it is now clearly recognized that the urothelium is not just a barrier, but it also plays an active role in neurogenic control of bladder function. ${ }^{5}$ The urothelium is supplied with afferent nerves and is involved in reflex responses to bladder filling and distension. In addition, it has a dense muscarinic receptor population and mediates the release of a diffusible factor that inhibits smooth muscle contraction. The pathophysiologic role of the urothelium is explored in greater detail by Dr. Pradeep Tyagi in this publication. 


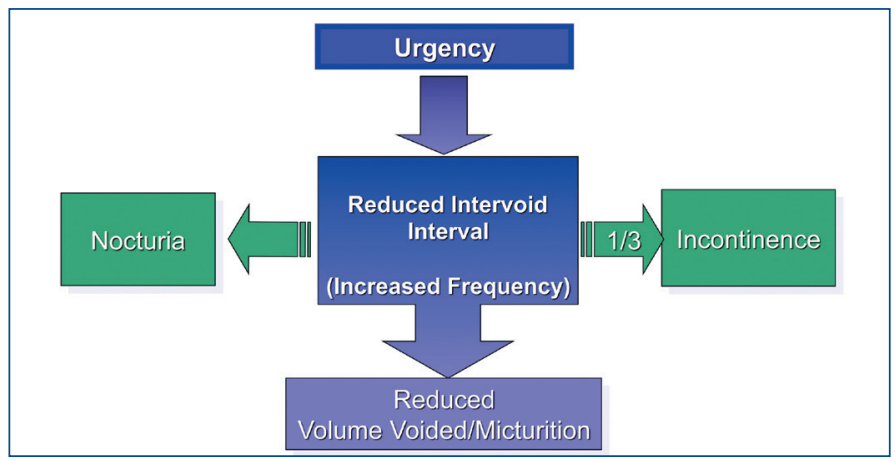

Fig. 1. Overactive bladder syndrome: a symptomatic sequence. Adapted from Chapple et al. ${ }^{6}$

\section{Implications for treatment}

Given that multiple efferent and afferent pathways are involved in LUT infection, each of these is a potential pharmacologic target for patients with $\mathrm{OAB}$. Antimuscarinics are still the mainstay of $O A B$ treatment. While there is no question that blockade of muscarinic receptors reduces detrusor muscle contractions, there may also be central and/or local neurologic effects of muscarinic blockade.

Other agents with activity on relevant neurotransmitters (e.g., antidepressants, antidiuretic hormone analogues, phosphodiesterase 5 inhibitors) may also have a role to play in treating symptoms of the LUT. Both sensory and motor pathways are potential future targets for pharmacologic intervention.

Capsaicin and resiniferotoxin are also under investigation for urinary tract symptoms. These agents bind to the vanilloid receptor in the urothelium and/or afferent pathway and produce neural damage, leading to impairment of bladder function and even retention. Botulinum toxin A and $B 3$-agonist therapy are also under investigation for the treatment of $\mathrm{OAB}$.

\section{Conclusions}

The pathophysiology of $\mathrm{OAB}$ is multifaceted. The common symptoms associated with this syndrome (e.g., urgency) seem to be mediated by a combination of myogenic and neurogenic mechanisms. Within the neurologic sphere, motor and sensory, central and peripheral, afferent and effer-

\begin{tabular}{|c|c|c|}
\hline Type & Location & Specific Aspects \\
\hline \multirow{2}{*}{ Sensory } & Peripheral & Increased afferent receptor excitability \\
\hline & Central & Abnormal central processing of afferent input \\
\hline \multirow[t]{2}{*}{ Motor } & Peripheral & $\begin{array}{l}\text { Smooth muscle contractile activity induced } \\
\text { by neural or non-neural mechanisms }\end{array}$ \\
\hline & Central & Involuntary parasympathetic nerve reflexes \\
\hline Mixed & Peripheral & $\begin{array}{l}\text { Leak of urine into proximal urethra and } \\
\text { stimulation of urethral afferents }\end{array}$ \\
\hline
\end{tabular}

ent aspects come into play. These multiple pathways provide a plethora of potential targets for pharmacologic modulation for the management of OAB. Indeed, antimuscarinic agents, which are currently the gold standard treatment, may also exert some of their beneficial effects through afferent as well as efferent pathways. Many other potential therapies are also currently being investigated.

Competing interests: Dr. Chapple is a consultant and/or speaker/researcher for Allergan, Astellas, GlaxoSmithKline, Lilly, Pfizer and Recordati.

This paper has been peer-reviewed.

\section{References}

1. Chapple $C R$, Artibani $W$, Cardozo LD, et al. The role of urinary urgency and its measurement in the overactive bladder symptom syndrome: current concepts and future prospects. BJU Int 2005;95:335-40.

2. Fowler CJ, Griffiths D, de Groat WC. The neural control of micturition. Nat Rev Neurosci 2008;9:453-66.

3. Griffiths D, Tadic SD. Bladder control, urgency, and urge incontinence: evidence from functional brain imaging. Neurourol Urodyn 2008;27:466-74.

4. Tadic $S$, Griffiths D, Schaefer W, et al. Brain activity measured by functional magnetic resonance imaging (FMRI) is related to patient reports and clinical severity of urge urinary incontinence. Presented at the International Continence Society Annual Meeting 2008; abstract \#214.

5. Chess-Williams R. Muscarinic receptors of the urinary bladder: detrusor, urothelial and prejunctional. Auton Autacoid Pharmacol 2002;22:133-45.

6. Chapple C, Arribani W, Cardozo LD, et al. The role of urinary urgency and its measurement in the overactive bladder symptom syndrome: current concepts and future prospects. BJU Int 2005;95:335-40.

Correspondence: Dr. Christopher Chapple, Consultant Urological Surgeon, Sheffield Teaching Hospitals NHS Foundation Trust, Department of Urology, The Royal Hallamshire Hospital, Sheffield, UK; fax: +44 (0)114 2797841; c.r.chapple@sheffield.ac.uk 